\title{
Comparison of long-term outcomes between Off- Pump CABG and conventional CABG
}

\author{
Won Yong Lee ${ }^{1 *}$, Jung Hyun Lim², Kun IL Kim³, Hyoung Soo Kim ${ }^{1}$ \\ From World Society of Cardiothoracic Surgeons 25th Anniversary Congress, Edinburgh \\ Edinburgh, UK. 19-22 September 2015
}

\section{Background/Introduction}

There has been controversy surrounding the late outcomes of Off-Pump Coronary Artery Bypass (OPCAB).

\section{Aims/Objectives}

The aim of this study was to compare the early and long-term outcomes of OPCAB with those of conventional CABG (c-CABG).

\section{Method}

This retrospective study was based on data from 183 patients who underwent CABG between January, 2000 and December, 2005. OPCAB was performed in 102 patients and c-CABG in 81 . The mean follow-up duration was 107 months. The end-points of long-term results were overall death, freedom from cardiac death and major adverse cardiac events (MACE). We expressed the Kaplan-Meyer survival curve, and determined the independent predictors for risk factors of mortality using multi-variate analysis.

\section{Results}

Four patients in c-CABG group died of low cardiac output syndrome and CVA. There was no operative mortalities in OPCAB group $(\mathrm{p}=.023)$. Bleeding requiring reopening $(5: 1, \mathrm{p}=.05)$ and CVA $(3: 0, \mathrm{p}=.05)$ occurred more frequently in c-CABG group than OPCAB group. The completeness of follow-up was $83.8 \%$.

Late deaths occurred in 26 patients (11 [18.0\%] in c-CABG group, 15 [16.9\%] in OPCAB group). The causes of death were cancer, CVA, cardiac and sepsis.

Rerevascularization was performed more frequently in OPCAB group than in c-CABG group $(14: 5, \mathrm{p}=.297)$. Five-year overall survival, freedom from cardiac death and MACE in c-CABG and OPCAB groups were 90.2 vs $96.6 \%, 98.4$ vs $100 \%$, and 91.8 vs $85.4 \%$, respectively. Ten-year survival estimates were 82.0 vs $83.1 \%$, 96.7 vs $96.6 \%$, and 88.5 vs $83.1 \%$, respectively. There were no statistical significance between 2 groups' survival $(\mathrm{p}=.743$ in overall survival, $\mathrm{P}=.813$ in free from cardiac death, and $p=.305$ in free from MACE). Age was an independent predictor for mortalities $(\mathrm{p}=0.000)$.

\section{Discussion/Conclusion}

OPCAB showed the better operative mortality and complication rates, and the higher rerevascularization rate, compared with c-CABG. Nevertheless, the survival indices did not reveal the statistical significance between 2 groups.

\section{Authors' details \\ ${ }^{1}$ Cardiothoracic Surgery, Hallym University Sacred Heart Hospital, Anyang, 431-070, South Korea. ${ }^{2}$ Cardiothoracic Surgery, Chosun University Hospital, Gwangju, 501-717, South Korea. ${ }^{3}$ Cardiothoracic Surgery, Hallym University Kangnam Sacred Heart Hospital, 150-950, South Korea.}

Published: 16 December 2015

doi:10.1186/1749-8090-10-S1-A243

Cite this article as: Lee et al:: Comparison of long-term outcomes between Off-Pump CABG and conventional CABG. Journal of Cardiothoracic Surgery 2015 10(Suppl 1):A243. 